\title{
Inhibición de respuesta como restricción y cancelación en individuos con normopeso, sobrepeso y obesidad
}

\author{
Response inhibition as restraint and cancellation \\ in individuals with normal weight, overweight, and obesity
}

\author{
Karla Paola Jiménez Cruz, Karla Edith González Alcántara \\ y Cecilia Silva Gutiérrez ${ }^{l}$
}

Citación: Jiménez Cruz, K.P., González Alcántara, K.E. y Silva Gutiérrez, C. (2021). Inhibición de respuesta como restricción y cancelación en individuos con normopeso, sobrepeso y obesidad. Psicología y Salud, 31(1), 93-102. https://doi.org/10.25009/pys.v31i1.2679.

\section{RESUMEN}

La información sobre si la inhibición de la respuesta es una variable asociada al sobrepeso y la obesidad es poco concluyente y contradictoria. Para aclarar dicha relación, la presente investigación tuvo como objetivos indagar si hay diferencias entre individuos con normopeso, sobrepeso y obesidad en la ejecución de las dos tareas más utilizadas para evaluar inhibición de respuesta, así como observar si se producen resultados diferentes cuando la inhibición se mide como cancelación o como restricción de la respuesta. Se controlaron los niveles de depresión y ansiedad en una muestra no probabilística e intencional de 100 jóvenes (50 hombres y 50 mujeres) de entre 18 y 29 años, agrupados de acuerdo a su índice de masa corporal. Se aplicaron dos tareas para evaluar la inhibición de la respuesta: Go/No-Go (restricción) y Stop Signal (cancelación). Los resultados mostraron que los participantes con normopeso, sobrepeso y obesidad lograron puntuaciones similares en ambas tareas. Se concluye que las personas con sobrepeso y obesidad no presentan dificultades en la inhibición de respuestas ante estímulos neutros.

Palabras clave: Impulsividad; Índice de Masa Corporal; Go/No-Go; Stop Signal Task.

\begin{abstract}
Information on whether response inhibition is a variable associated with overweight and obesity is inconclusive and contradictory. To clarify this relationship, the present study aimed at documenting differences between individuals with normal weight, overweight, and obesity on performance in the two most used tasks to assess response inhibition. An additional objective sought to explore if different results occur when the inhibition is measured as cancellation or restriction of the response. Depression and anxiety levels were controlled in a probabilistic intentional sample of 100 young participants (50 men and 50 women) between 18 and 29 years old, grouped according to their body mass index. Two tasks were used to evaluate response inhibition: Go/No-Go task (restriction) and Stop Signal Task (cancellation). The findings show that normal weight, overweight and obese participants, present similar scores in both response inhibition tasks. It is concluded that people with overweight and obesity do not present difficulties in the inhibition response to neutral stimuli.
\end{abstract}

Keywords: Impulsivity; Body Mass Index; Go/No-Go; Stop Signal Task.

\footnotetext{
${ }^{1}$ Facultad de Psicología, Universidad Nacional Autónoma de México, Av. Universidad 3004, Edificio E, Cubículo 208, Del. Coyoacán, 04510 Ciudad de México, México, tel. (55)56-22-82-22, ext. 41161, correos electrónicos: karlapao.ola@gmail.com, karlaedith@comunidad.unam.mx y csilva@posgrado.unam.mx. Artículo recibido el 5 de diciembre de 2019 y aceptado el 30 de mayo de 2020.
} 
$\mathrm{E}$ 1 sobrepeso y la obesidad son los principales factores de riesgo para desarrollar enfermedades crónicas no transmisibles, y sus consecuencias a largo plazo son negativas e incluyen un deterioro de la salud emocional y comorbilidades médicas importantes (Organización Mundial de la Salud [OMS], 2020; Pasco, Williams, Jacka, Brennan y Berk, 2013), así como estigmatización social (Puhl, Luedicke y Heuer, 2013).

En México, los resultados del año 2018 de la Encuesta Nacional de Salud y Nutrición (ENSANUT) (Instituto Nacional de Estadística y Geografía [INEGI] e Instituto Nacional de Salud Pública [INSP], 2018) mostraron que la prevalencia de sobrepeso y obesidad en adultos de 20 años o más fue de $75.2 \%$, habiendo más hombres $(42.5 \%)$ que mujeres $(36.6 \%)$ con sobrepeso, pero más mujeres $(40.2 \%)$ que hombres $(30.5 \%)$ con obesidad, lo que coloca al país en el segundo lugar de obesidad en el mundo, solo superado por Estados Unidos (Organization for Economic Cooperation and Development [OECD], 2017), por lo que es necesario conocer los factores que contribuyen a su aparición.

Aunque el sobrepeso y la obesidad se deben a un alto consumo calórico (OMS, 2020), es posible que la conducta de sobreingesta de calorías esté asociada a una deficiencia del control inhibitorio (Smith, Hay, Campbell y Trollor, 2011), que se refiere a la habilidad para suprimir acciones o respuestas inapropiadas o que no son necesarias (Bartholdy, Dalton, O'Daly, Campbell y Schmidt, 2016; Chambers, Garavan y Bellgrove, 2009).

Tal posibilidad se ha planteado en cuanto que un control inhibitorio deficiente dificulta restringir conductas en general, y no solo las relacionadas con el consumo excesivo de alimentos (Bari y Robbins, 2013). Si fuera así, una inhibición deficiente podría desempeñar un importante papel en el mantenimiento del sobrepeso y la obesidad (Bartholdy et al., 2016; Batterink, Yokum y Stice, 2010; Chambers et al., 2009; Volkow, Wang y Baler, 2011).

Algunas investigaciones reportan que hay relación entre la inhibición y la obesidad. Por ejemplo, Mobbs, Iglesias, Golay y Van der Linden (2011) al evaluar con la tarea Go/No-Go (que contenía estímulos vinculados con la comida y el cuerpo) a un grupo de adultos con obesidad (hombres y mujeres de alrededor de 40 años) encontra- ron que este tenía más dificultades en los procesos de inhibición que otro de adultos con normopeso. También Houben, Nederkoorn y Jansen (2014) reportan una relación negativa entre el Índice de Masa Corporal (IMC) y la inhibición de respuesta al evaluarla en mujeres con una media de edad de 26.17 años mediante la tarea Stop Signal Task (SST en lo sucesivo), conformada por estímulos relacionados con la comida.

Adicionalmente, los resultados de Chamberlain, Derbyshire, Leppink y Grant (2015) en la SST mostraron que hombres y mujeres de 18 a 29 años con obesidad obtuvieron puntajes más altos que individuos con normopeso, incluso ante estímulos neutros. Nederkoorn, Smulders, Havermans, Roefs y Jansen (2006), a su vez, valoraron a mujeres de aproximadamente 40 años, observando respuestas de inhibición menos efectivas en la última parte de la tarea SST cuando presentaban obesidad, lo que sugiere que podrían tener dificultades para mantener el control inhibitorio aunque no mostraran un deterioro general.

Sin embargo, en otras investigaciones no se halla tal relación. Por ejemplo, Fields, Sabet y Reynolds (2013), al evaluar la inhibición de respuesta con una tarea tipo SST, no encontraron diferencias entre adolescentes de 14 a 16 años (hombres y mujeres) con normopeso y con obesidad. Tampoco Lawyer, Boomhower y Rasmussen (2015) observaron diferencias en la inhibición en una SST entre personas (hombres y mujeres de 18 a 30 años) con y sin obesidad. Loeber et al. (2012) tampoco encontraron diferencias significativas entre hombres y mujeres de 18 a 65 años empleando una prueba Go/No-Go, e incluso los resultados de un metaanálisis de Lavagnino et al. (2016) demuestran que si bien algunos datos muestran que hay deficiencias en la inhibición en personas con obesidad, no son comunes a todos los estudios.

Tal incongruencia en los resultados podría atribuirse a diferentes factores que no han sido considerados en las investigaciones previas. Por ejemplo, se han utilizado indiferenciadamente tareas para evaluar el control inhibitorio no obstante que evalúan componentes distintos de la inhibición. La tarea Go/No-Go mide la inhibición de una respuesta planificada -es decir, la restricción de una fuerte tendencia a responder ante una señal que indica no hacerlo-, en tanto que la SST valora 
la inhibición de una acción ya iniciada - o sea, la cancelación de una respuesta continua cuando se presenta una señal que indica no responder (Schachar et al., 2007; Winstanley, 2011); por lo tanto, las tareas involucran componentes diferentes de la inhibición.

Además, no se ha tenido control sobre estados afectivos como la ansiedad y la depresión, que son reportados consistentemente como variables que presentan comorbilidad con la obesidad (De Wit et al., 2010; Gariepy, Nitka, y Schmitz, 2010) y que podrían influir en los resultados al afectar en la ejecución de las tareas (Ardila y Ostrosky, 2012). Asimismo, algunas investigaciones se han centrado en evaluar solo a mujeres, o bien analizan a hombres y mujeres como un grupo homogéneo, cuando se sabe que en lo relativo al control inhibitorio hay diferencias entre los sexos (Yuan, He, Qinglin, Chen y Li, 2008), y finalmente la literatura previa ha evaluado poblaciones con rangos de edad muy amplios, lo cual podría afectar asimismo los resultados.

Por tal motivo, y dado que las investigaciones publicadas no son concluyentes, el objetivo del presente estudio fue conocer si hay diferencias entre individuos con normopeso, sobrepeso y obesidad en la ejecución de las dos tareas más utilizadas para evaluar inhibición de respuesta, y observar si se producen resultados diferentes cuando la inhibición se mide como cancelación o como restricción de la respuesta. Para ello, se tomaron en cuenta las variables que pueden afectar el control inhibitorio y que no se han controlado en los estudios previos: los niveles de depresión y ansiedad, la edad y el sexo, de modo que la información obtenida podría enriquecer los resultados de estudios previos y contribuir al esclarecimiento de la relación que el control inhibitorio mantiene con el sobrepeso y la obesidad.

\section{MÉTODO}

\section{Participantes}

Para este estudio se evaluó inicialmente a 305 adultos jóvenes; sin embargo, para evitar que los niveles de ansiedad y depresión pudieran influir en los resultados al afectar la ejecución de las tareas, se excluyeron los casos que mostraban niveles severos de ansiedad o depresión, medidas con el Inventario de Ansiedad de Beck (puntuación total $\geq 31$ ) y el Inventario de Depresión de Beck II (puntuación total $\geq 29$ ), respectivamente.

De igual forma se eliminaron los datos de los participantes con un IMC menor o igual a 18.4 al caer en la categoría de bajo peso (World Health Organization [WHO], 2000). Por último, dado que estudios previos sobre la inhibición de respuesta y la obesidad han evaluado a grupos con rasgos de edad muy amplios y tratado a los participantes de ambos sexos como si formaran parte de un mismo grupo, lo cual impide comparar los resultados, se decidió igualar a los participantes en cuanto a su edad ( \pm 1 año) y sexo.

Así, los análisis se realizaron con los datos de 100 adultos jóvenes residentes de la Ciudad de México (50 hombres y 50 mujeres), con edades de 18 a 29 años, con IMC comprendido entre 18.59 y 39.04 (Tabla 1), que se categorizaron en tres grupos de peso: normopeso (IMC entre 18.5 y 24.9), sobrepeso (IMC entre 25 y 29.9) y obesidad (IMC mayor o igual a 30) (WHO, 2000). En la Tabla 1 se describen las características de los participantes.

Tabla 1. Datos generales y número de participantes por categoría de peso.

\begin{tabular}{|l|c|c|c|c|c|c|}
\hline \multirow{2}{*}{ Datos generales } & \multicolumn{2}{|c|}{$\begin{array}{c}\text { Hombres } \\
\mathbf{n}=\mathbf{5 0}\end{array}$} & \multicolumn{2}{c|}{$\begin{array}{c}\text { Mujeres } \\
\mathbf{n = 5 0}\end{array}$} & \multicolumn{2}{c|}{$\begin{array}{c}\text { Total } \\
\mathbf{N}=\mathbf{1 0 0}\end{array}$} \\
\cline { 2 - 7 } & $\mathbf{M}$ & $\mathbf{D . E}$. & $\mathbf{M}$ & $\mathbf{D . E}$. & $\mathbf{M}$ & $\mathbf{D . E}$. \\
\hline Edad & 22.18 & 3.35 & 21.20 & 2.94 & 21.69 & 3.18 \\
\hline IMC & 26.64 & 4.63 & 26.19 & 4.42 & 26.42 & 4.51 \\
\hline \multicolumn{6}{|c|}{ Categorías por peso } \\
\hline Normopeso & \multicolumn{2}{|c|}{$\mathrm{n}=21$} & $\mathrm{n}=21$ & $\mathrm{n}=42$ \\
\hline Sobrepeso & $\mathrm{n}=14$ & $\mathrm{n}=20$ & $\mathrm{n}=34$ \\
\hline Obesidad & $\mathrm{n}=15$ & $\mathrm{n}=9$ & $\mathrm{n}=24$ \\
\hline
\end{tabular}

Nota $:$ IMC = Índice de Masa Corporal; $\mathrm{M}=$ Media; D.E. = Desviación estándar. 


\section{Instrumentos y tareas}

Se emplearon las adaptaciones para población mexicana del Inventario de Ansiedad de Beck (Robles, Varela, Jurado y Páez, 2001) y del Inventario de Depresión de Beck II (González, Reséndiz y Reyes, 2015) para evaluar la ansiedad y la depresión, respectivamente, y excluir a los individuos con niveles severos en estas dos variables.

Adicionalmente se incluyó un cuestionario de datos sociodemográficos del que se obtuvieron la edad, el sexo, la escolaridad, y el autorreporte de peso y talla para calcular el IMC (puesto que se ha visto que hay una buena relación entre dicho autorreporte y las medidas reales de las personas) (Osuna, Hernández, Campuzano y Salmerón, 2006).

La batería, a la vez, estuvo conformada por dos tareas para evaluar la inhibición de respuesta: Go/No-Go Task, que evalúa la inhibición como restricción (Fillmore, Rush y Hays, 2006), y la Stop Signal Task, que mide la inhibición como cancelación (Logan, Schachar y Tannock, 1997).

\section{Go/No-Go Task}

La tarea Go/No-Go involucra situaciones en las que una dificultad en el control inhibitorio promueve la comisión de errores debido a que el participante debe dar una respuesta motora ante la presentación de ciertos estímulos, pero debe frenarla ante otros (Noreña y Sánchez, 2015). Esta tarea mide la inhibición de una respuesta planificada o, en otras palabras, la restricción de la tendencia a responder ante una señal que indica no hacerlo (Schachar et al., 2007). Utiliza dos señales: Go, que indica que se requiere emitir una respuesta, y No-Go, que señala que debe inhibirse la respuesta (Winstanley, 2011).

La tarea consistió en presentar a los participantes una serie de ensayos, solicitándoles que presionaran la barra espaciadora del teclado tan pronto como apareciera un rectángulo verde en la pantalla (señal Go), pero abstenerse de presionarla cuando fuera un rectángulo azul (señal No-Go). En cada ensayo, los rectángulos verde o azul tenían una orientación vertical u horizontal; mientras que los rectángulos verticales tenían una probabilidad de $80 \%$ de ser verdes y $20 \%$ de ser azules, los horizontales tenían, a su vez, una probabilidad de $80 \%$ de ser azules y $20 \%$ de ser verdes.

La orientación funcionó como una pista que señala la probabilidad de que se presente una señal Go o una No-Go; entre el inicio de una pista y el comienzo de una señal hay un cierto tiempo que asegura la asincronía de la presentación de estímulos.

La orientación vertical -pista para la señal Go- generaba respuestas predominantes que aceleraban el tiempo de reacción, ante lo cual los participantes debían restringir la respuesta predominante con el fin de inhibirla si se presenta una señal No-Go.

Cada ensayo consistió de lo siguiente: $a$ ) presentación de un punto de fijación por $800 \mathrm{~ms}$; b) una pantalla en blanco por $500 \mathrm{~ms}$; c) una pista (rectángulo vertical u horizontal) para una de las cinco asincronías de la presentación de estímulos $(100,200,300,400$ o $500 \mathrm{~ms})$; d) una señal Go (color verde) o No-Go (azul), que permanecía visible hasta que el participante respondía después de transcurridos $1000 \mathrm{~ms}$, y e) un intervalo entre ensayos de $700 \mathrm{~ms}$.

En cada ensayo se presentó una combinación entre pistas (vertical u horizontal) y señales (Go o No-Go), siendo aleatoria la presentación de estas combinaciones. Se aplicaron 50 ensayos en los que se cumplieron todas las condiciones y proporciones.

En esta tarea, la cantidad de errores (responder ante una señal No-Go cuando se presenta una pista para Go) indicaba si el participante lograba o no inhibir su respuesta (Fillmore et al., 2006).

\section{Stop Signal Task (SST)}

Consiste en una serie de ensayos en los que aparecen estímulos ante los que se responde de manera continua, y de otros ensayos en la que se presenta una señal Stop que indica que no se debe responder, por lo que se debe inhibir la respuesta ya planificada (Logan et al., 1997).

La tarea mide la inhibición de una acción ya iniciada, es decir, implica la cancelación de una respuesta continua cuando se presenta una señal que indica no responder, como un sonido (Schachar et al., 2007). 
La tarea consta de dos fases: una de práctica, con un bloque de 32 ensayos, y una de prueba, con tres bloques de 64 ensayos cada uno. En el presente trabajo, en ambas fases, cada ensayo constaba de lo siguiente: a) la presentación de un punto de fijación (un círculo blanco) en el centro de la pantalla en negro; $b$ ) después de $250 \mathrm{~ms}$, aparecía el estímulo primario (una flecha horizontal blanca); c) la flecha permanecía en la pantalla hasta que el participante respondía, o bien después de $1250 \mathrm{~ms}$, que es el máximo tiempo de reacción que asigna el programa; d) había un intervalo de 2000 $\mathrm{ms}$ antes de que comenzara el siguiente ensayo.

Los estímulos eran flechas que podían apuntar a la derecha o la izquierda, y el participante debía presionar la tecla D si la flecha apuntaba hacia la izquierda, o la tecla K si apuntaba a la derecha, siempre y cuando no se mostrara la señal Stop (un sonido) una vez que la flecha aparecía. En caso de que se presentara el sonido, la respuesta planificada debía cancelarse.

En los ensayos con señal Stop, el sonido era presentado después de la flecha con un retraso variable de la señal Stop (SSD, por sus siglas en inglés). El SSD inicial fue de $250 \mathrm{~ms}$ y se ajustaba continuamente mediante un proceso de seguimiento: cuando la inhibición era exitosa, el SSD aumentaba $50 \mathrm{~ms}$; cuando no lo era, el SSD decrecía $50 \mathrm{~ms}$. Entre mayor fuera el SSD, más difícil era inhibir la respuesta planeada (Verbruggen, Logan y Stevens, 2008; Winstanley, 2011).

En esta tarea, el tiempo de reacción para la señal Stop (SSRT, por sus siglas en inglés) es el indicador de inhibición de respuesta: a medida que el SSRT es mayor, hay menos capacidad para inhibir la respuesta (Fillmore et al., 2006; Logan et al., 1997; Verbruggen et al., 2008; Winstanley, 2011).

\section{Inventario de Ansiedad de Beck (IAB)}

(Beck, Epstein, Brown y Steer, 1988).

Adaptado para población mexicana por Robles et al. (2001), es uno de los instrumentos más utilizados para evaluar la sintomatología ansiosa, ya que permite detectar síntomas en la población general y clínica.

Consta de 21 reactivos en una escala tipo Likert con cuatro opciones de respuesta, que van de 0 (poco o nada) a 3 (severamente). Con base en el puntaje total puede clasificarse la sintomatología ansiosa del participante como mínima (0-5), leve (6-15), moderada (16-30) o severa (31-63). Este instrumento se utilizó en este trabajo para excluir de los análisis a los participantes con ansiedad severa.

\section{Inventario de Depresión de Beck II (IDB-II)}

(Beck, Steer y Brown, 1996).

Originalmente elaborado por Beck, Ward, Mendelson, Mock y Erbaugh (1961), es uno de los instrumentos autoaplicables más utilizados para evaluar los síntomas de depresión. En 1996, Beck, Steer y Brown publicaron una nueva versión, el IDB-II, misma que fue adaptada a estudiantes y población general por González et al. (2015).

El instrumento consta de 21 ítems en una escala tipo Likert de cuatro puntos, que va de 0 a 3 ; mayores puntajes indican mayor intensidad de la sintomatología depresiva. Con base en los puntos de corte, la puntuación total obtenida permite clasificar en depresión mínima (0-13), leve (14-19), moderada (20-28) o severa (29-63). En la presente investigación se le empleó para excluir de los análisis a los participantes con depresión severa.

\section{Procedimiento}

Las evaluaciones se llevaron a cabo vía internet por medio de la plataforma Inquisit Web, versión 4, por Millisecond, un proveedor de software que permite aplicar tareas neuropsicológicas, instrumentos psicométricos, cuestionarios a distancia y datos en línea mediante una computadora.

Inicialmente, en el Inquisit Web se conformó con la batería aplicada, que constaba de un formato de consentimiento informado (elaborado de acuerdo con las políticas regulatorias de la declaración de Helsinki en cuanto al cumplimiento de los principios éticos de participación voluntaria y gestión de datos confidenciales), el cuestionario de datos sociodemográficos, el IAB, el IDB, la tarea Go/No-Go y la tarea Stop Signal Task. El tiempo aproximado para contestar la batería fue de 20 minutos.

Una vez conformada la batería en la plataforma, a través de las redes sociales y en los propios salones de clase se invitó a participar a los 
alumnos de una universidad pública y de una privada. En ambos casos se describían los objetivos de la investigación, y a quienes se mostraran interesados se les indicaba el vínculo que los dirigiría a la plataforma en la que se encontraba la batería de pruebas.

Los participantes podían acceder al vínculo únicamente desde una computadora conectada a internet ${ }^{2} \mathrm{y}$ en el horario que desearan.

Cuando accedían al vínculo primero se les presentaba el formato de consentimiento informado; si decidían participar, podían continuar respondiendo los siguientes apartados de la batería; si no, podían salir de la plataforma en ese momento. Al finalizar, se les proporcionaba un correo electrónico al que podían escribir dudas o comentarios. Luego, Inquisit Web envió los datos obtenidos de cada participante a una base, la cual se descargó de la plataforma, y después transferida a una base de datos de SPSS para su análisis estadístico.

Inicialmente se probaron los supuestos para pruebas paramétricas con el propósito de conocer si las variables de interés tenían una distribución normal y si había homogeneidad de varianza entre grupos. Tras no cumplir con esos supuestos, se hicieron pruebas Kruskal-Wallis para conocer las diferencias entre los grupos con diferentes IMC y entre mujeres y hombres.

\section{RESULTADOS}

\section{Diferencias entre participantes con normopeso, sobrepeso y obesidad}

Los resultados muestran que no hubo diferencias estadísticamente significativas al evaluar la inhibición de respuesta como restricción ni como cancelación (Tabla 2), y que las personas con exceso de peso no difirieron de aquellas con normopeso, independientemente de la tarea empleada; es decir, si la inhibición se evaluaba como la restricción de una respuesta planificada o como la cancelación de una acción ya iniciada.

\section{Diferencias entre mujeres y hombres con nor- mopeso, sobrepeso u obesidad}

Al comparar a los hombres y mujeres con normopeso, sobrepeso y obesidad, tampoco se hallaron diferencias estadísticamente significativas en la inhibición de respuesta en ninguna de las tareas empleadas (Tabla 3).

Tabla 2. Diferencias entre las personas con peso normal, sobrepeso y obesidad.

\begin{tabular}{|c|c|c|c|c|c|}
\hline \multirow{3}{*}{ Variable } & \multirow{3}{*}{ Grupos } & \multicolumn{4}{|c|}{ Prueba de Kruskal-Wallis } \\
\hline & & \multirow{2}{*}{$\begin{array}{l}\text { Rango } \\
\text { promedio }\end{array}$} & \multirow{2}{*}{ Mediana } & \multicolumn{2}{|c|}{$\begin{array}{l}\text { Estadísticos } \\
\text { de contraste }\end{array}$} \\
\hline & & & & $x^{2}$ & $p$ \\
\hline \multicolumn{6}{|c|}{ Tarea Go/No-Go } \\
\hline \multirow{3}{*}{$\begin{array}{l}\text { Tasa de error para ensayos con pista } \mathrm{V} \\
\text { y estímulo NG }\end{array}$} & Normopeso & 50.18 & 0.00 & \multirow{3}{*}{0.62} & \multirow{3}{*}{0.73} \\
\hline & Sobrepeso & 49.10 & 0.00 & & \\
\hline & Obesidad & 53.04 & 0.00 & & \\
\hline \multicolumn{6}{|c|}{ Tarea SST } \\
\hline \multirow{3}{*}{$\begin{array}{l}\text { Estimación del tiempo de reacción } \\
\text { para la señal stop (SSRT) }\end{array}$} & Normopeso & 48.31 & 257.09 & \multirow{3}{*}{2.55} & \multirow{3}{*}{0.27} \\
\hline & Sobrepeso & 56.76 & 264.89 & & \\
\hline & Obesidad & 45.46 & 250.99 & & \\
\hline
\end{tabular}

Nota $: \mathrm{V}=$ Vertical; $\mathrm{NG}=$ No-Go; $\mathrm{SSRT}=$ Stop Signal Reaction Time; $\mathrm{SST}=$ Stop Signal Task.

\footnotetext{
${ }^{2}$ Las características del software de Inquist impiden acceder desde una tableta o un teléfono celular.
} 
Tabla 3. Diferencias entre mujeres y hombres con normopeso, sobrepeso u obesidad.

\begin{tabular}{|c|c|c|c|c|c|}
\hline \multirow{3}{*}{ Variable } & \multirow{3}{*}{ Grupo } & \multicolumn{4}{|c|}{ Prueba de Kruskal-Wallis } \\
\hline & & \multirow{2}{*}{$\begin{array}{c}\text { Rango } \\
\text { promedio }\end{array}$} & \multirow[t]{2}{*}{ Mediana } & \multicolumn{2}{|c|}{$\begin{array}{l}\text { Estadísticos } \\
\text { de contraste }\end{array}$} \\
\hline & & & & $x^{2}$ & $p$ \\
\hline \multicolumn{6}{|c|}{ Tarea Go/No-Go } \\
\hline \multirow{6}{*}{$\begin{array}{l}\text { Tasa de error para ensayos con pista } \mathrm{V} \\
\text { y estímulo NG }\end{array}$} & Mujer normopeso & 51.31 & 0.00 & \multirow{6}{*}{1.76} & \multirow{6}{*}{0.88} \\
\hline & Mujer sobrepeso & 51.78 & 0.00 & & \\
\hline & Mujer obesidad & 53.78 & 0.00 & & \\
\hline & Hombre normopeso & 49.05 & 0.00 & & \\
\hline & Hombre sobrepeso & 45.29 & 0.00 & & \\
\hline & Hombre obesidad & 52.60 & 0.00 & & \\
\hline \multicolumn{6}{|c|}{ Tarea SST } \\
\hline \multirow{6}{*}{$\begin{array}{l}\text { Estimación del tiempo de reacción } \\
\text { para la señal Stop (SSRT) }\end{array}$} & Mujer normopeso & 50.38 & 259.44 & \multirow{6}{*}{4.82} & \multirow{6}{*}{0.43} \\
\hline & Mujer sobrepeso & 50.80 & 261.10 & & \\
\hline & Mujer obesidad & 44.67 & 244.07 & & \\
\hline & Hombre normopeso & 46.24 & 244.00 & & \\
\hline & Hombre sobrepeso & 65.29 & 267.02 & & \\
\hline & Hombre obesidad & 45.93 & 257.90 & & \\
\hline
\end{tabular}

Nota $: \mathrm{v}=$ Vertical; $\mathrm{NG}=$ No-Go; SSRT $=$ Stop Signal Reaction Time; SST $=$ Stop Signal Task.

\section{DISCUSIÓN}

En este estudio no se encontraron diferencias en la inhibición de respuesta entre los participantes con peso normal, sobrepeso y obesidad en la restricción de una respuesta planificada ni en la cancelación de una acción iniciada.

Los datos obtenidos son congruentes con los de otros trabajos que tampoco encontraron una asociación entre la obesidad y una baja capacidad de inhibición de repuesta como restricción (Loeber et al. 2012) ni como cancelación (Fields et al., 2013; Lawyer et al., 2015), pero no coinciden con los de otras investigaciones que la han evaluado como cancelación (Chamberlain et al., 2015; Houben et al., 2014; Nederkoorn et al., 2006) y como restricción (Mobbs et al., 2011), las cuales han hallado diferencias en la inhibición entre personas obesas y con normopeso.

La comparación de la ejecución de mujeres y hombres con normopeso, sobrepeso y obesidad no mostró diferencias significativas en la inhibición de respuesta en ninguna de las tareas empleadas. Cabe mencionar que no es del conocimiento de las presentes autoras algún estudio que evalúe las diferencias en la inhibición de respuesta com- parando hombres y mujeres con diferentes IMC, toda vez que en la mayoría de estos se ha evaluado solo a mujeres, o bien a hombres y mujeres como un grupo homogéneo, sin hacer por consiguiente comparaciones entre sexos.

El que no se hayan encontrado diferencias entre los grupos se puede deber a diferentes razones que vale la pena considerar para proseguir la investigación; por ejemplo, el tipo de inhibición de respuesta que se ha abordado tanto en esta investigación como en los estudios previos, así como los tipos de estímulos contenidos en las tareas. En cuanto a lo primero, las tareas SST y Go/No-Go evalúan la inhibición motora, que se refiere a la capacidad para suprimir comportamientos no necesarios o inapropiados (Chambers et al., 2009). Sin embargo, no es el único tipo de inhibición de respuesta ya que también existe la inhibición de respuesta de índole afectiva (Verdejo y Bechara, 2010), basada en la dificultad para demorar la obtención de gratificación o recompensas y que puede valorarse mediante paradigmas de descuento temporal (Bari y Robbins, 2013).

Cuando se han estimado las deficiencias en la inhibición afectiva se ha observado que muestra cierta asociación con el sobrepeso (Fields et al., 
2013; Lawyer et al., 2015). Es posible entonces, que no sea la inhibición de respuesta motora la que esté relacionada con el exceso de peso sino la afectiva, pero esto es solamente una conjetura que habría que corroborar.

Por otro lado, se ha planteado que los tipos de estímulos empleados en las tareas desempeñan un papel importante en los resultados obtenidos, y que es posible que únicamente se observen deficiencias en el control inhibitorio motor en las personas obesas cuando las tareas se conforman con estímulos relacionados con alimentos (Bartholdy et al., 2016; Houben et al., 2014). Sin embargo, Loeber et al. (2012) hallaron que los participantes obesos y los normopeso muestran índices de inhibición de respuesta similares en las tareas con estímulos neutros y en aquellas con estímulos relacionados con alimentos, por lo que los datos sobre el tipo de estímulos tampoco son concluyentes. También vale la pena considerar la posibilidad de que la habilidad para inhibir algunos comportamientos ocurra solo cuando el individuo intenta llevar una dieta para bajar de peso o para mantener un peso saludable (Lavagnino et al., 2016).

El aporte principal de esta investigación es que incluso al emplear las dos tareas más utilizadas para evaluar la inhibición de respuestas y controlar variables tales como los niveles de ansiedad y depresión severos, el sexo y la edad, no se observaron asociaciones entre la inhibición de respuesta motora y el sobrepeso en población mexicana, específicamente; es importante señalar que las investigaciones revisadas se llevaron a cabo en Europa y Estados Unidos. Así, estos resultados constituyen solamente un primer acercamiento al fenómeno, eliminando la influencia de aquellas variables que pudieran afectarlo. Sin embargo, se debe tomar en cuenta que los análisis se realizaron en grupos pequeños que no mostraban una distribución normal.

En futuras investigaciones sería recomendable incluir un grupo de personas con el llamado trastorno por atracón, ya que es probable que sea una variable que influya en los resultados, y que por ello estos sean contradictorios. También habría que considerar aplicar tareas que no únicamente contengan estímulos neutros, e incluir también estímulos relacionados con la comida y la alimentación, a fin de saber si la capacidad de inhibición es diferente con cada tipo de estímulo.

Los resultados del presente estudio se deben tomar con cautela ya que, por un lado, los datos no se distribuyeron normalmente, por lo que no son generalizables a la población y, por otro lado, aunque las aplicaciones en línea tienen la ventaja de que eliminan el error en la calificación y la conformación de bases de datos, también elevan la probabilidad de que factores externos intervengan en la ejecución.

En conclusión, en este estudio se corroboró que la inhibición de respuesta motora (de restricción y de cancelación) no se relaciona con el sobrepeso ni con la obesidad cuando se emplean estímulos neutros, pues no hubo diferencias en la ejecución de las tareas entre los individuos con normopeso, sobrepeso y obesidad, independientemente del tipo de tarea utilizada, incluso controlando los niveles de ansiedad y de depresión de los participantes, ni tampoco se observaron diferencias entre hombres y mujeres.

Finalmente, con base en la literatura revisada, es posible que el sobrepeso y la obesidad se relacionan con el descuento temporal, pero no con la incapacidad para inhibir conductas.

\section{REFERENCIAS}

Ardila, A. y Ostrosky S., F. (2012). Guía para el diagnóstico neuropsicológico. México: UNAM.

Bari, A. y Robbins, T.W. (2013). Inhibition and impulsivity: behavioral and neural basis of response control. Progress in Neurobiology, 108(1), 44-79. Doi: 10.1016/j.pneurobio.2013.06.005.

Bartholdy, S., Dalton, B., O’Daly, O.G., Campbell, I.C. y Schmidt, U. (2016). A systematic review of the relationship between eating, weight and inhibitory control using the stop signal task. Neuroscience \& Biobehavioral Reviews, 64, 35-62. Doi: 10.1016/j.neubiorev.2016.02.010.

Batterink, L., Yokum, S. y Stice, E. (2010). Body mass correlates inversely with inhibitory control in response to food among adolescent girls: an fMRI study. Neuroimage, 52(4), 1696-1703. Doi: 10.1016/j.neuroimage.2010.05.059. 
Beck, A.T., Epstein, N., Brown, G.K. y Steer, R.A. (1988). An inventory for measuring clinical anxiety psychometric properties. Journal of Consulting and Clinical Psychology, 56(6), 893-897.

Beck, A.T., Steer, R.A. y Brown, G.K. (1996). BDI-II. Beck Depression Inventory (2nd ed.). San Antonio, TX: The Psychological Corporation.

Beck, A.T., Ward, C.H., Mendelson, M., Mock, J. y Erbaugh, J. (1961). An inventory for measuring depression. Archives of General Psychiatry, 4, 561-571.

Chamberlain, S.R., Derbyshire, K.L., Leppink, E. y Grant, J.E. (2015). Obesity and dissociable forms of impulsivity in young adults. CNS Spectrums, 20(5), 500-507. Doi: 10.1017/S1092852914000625.

Chambers, C.D., Garavan, H. y Bellgrove, M.A. (2009). Insights into the neural basis of response inhibition from cognitive and clinical neuroscience. Neuroscience \& Biobehavioral Reviews, 33(5), 631-646. Doi: 10.1016/j.neubiorev.2008.08.016.

De Wit, L.M., Fokkema, M., Van Straten, A., Lamers, F., Cuijpers, P. y Penninx, B.W.J.H. (2010). Depressive and anxiety disorders and the association with obesity, physical, and social activities. Depression and Anxiety, 27(11), 1057-1065. Doi: 10.1002/da.20738.

Fields, S.A., Sabet, M. y Reynolds, B. (2013). Dimensions of impulsive behavior in obese, overweight, and healthy-weight adolescents. Appetite, 70(1), 60-66. Doi: 10.1016/j.appet.2013.06.089.

Fillmore, M.T., Rush, C.R. y Hays, L. (2006). Acute effects of cocaine in two models of inhibitory control: implications of non-linear dose effects. Addiction, 101(9), 1323-1332. Doi: 10.1111/j.1360-0443.2006.01522.x.

Gariepy, G., Nitka, D. y Schmitz, N. (2010). The association between obesity and anxiety disorders in the population: a systematic review and meta-analysis. International Journal of Obesity, 34(3), 407-419. Doi: 10.1038/ijo.2009.252.

González D., A., Reséndiz, A. y Reyes L., I. (2015). Adaptación del IDB-II en México. Salud Mental, 38(4), 237-244. Doi: 10.17711/SM.0185-3325.2015.033.

Houben, K., Nederkoorn, C. y Jansen, A. (2014). Eating on impulse: The relation between overweight and food-specific inhibitory control. Obesity, 22(5), E6-E8. Doi: 10.1002/oby.20670.

Instituto Nacional de Estadística y Geografía e Instituto Nacional de Salud Pública (2018). Encuesta Nacional de Salud y Nutrición 2018: Presentación de resultados. Cuernavaca (México): INSP. Recuperado de https://ensanut.insp.mx/encuestas/ ensanut2018/doctos/informes/ensanut_2018_presentacion_resultados.pdf.

Lavagnino, L., Arnone, D., Cao, B., Soares, J.C. y Selvaraj, S. (2016). Inhibitory control in obesity and binge eating disorder: A systematic review and meta-analysis of neurocognitive and neuroimaging studies. Neuroscience \& Biobehavioral Reviews, 68, 714-726. Doi: 10.1016/j.neubiorev.2016.06.041.

Lawyer, S.R., Boomhower, S.R. y Rasmussen, E.B. (2015). Differential associations between obesity and behavioral measures of impulsivity. Appetite, 95, 375-382. Doi: 10.1016/j.appet.2015.07.031.

Loeber, S., Grosshans, M., Korucuoglu, O., Vollmert, C., Vollstädt-Klein, S., Schneider, S. y Kiefer, F. (2012). Impairment of inhibitory control in response to food-associated cues and attentional bias of obese participants and normal-weight controls. International Journal of Obesity, 36(10), 1334-1339. Doi: 10.1038/ijo.2011.184.

Logan, G.D., Schachar, R.J. y Tannock, R. (1997). Impulsivity and inhibitory control. Psychological Science, 8(1), 60-64.

Mobbs, O., Iglesias, K., Golay, A. y Van der Linden, M. (2011). Cognitive deficits in obese persons with and without binge eating disorder. Investigation using a mental flexibility task. Appetite, 57(2011), 263-271. Doi: 10.1016/j.appet.2011.04.023.

Nederkoorn, C., Smulders, F.T., Havermans, R.C., Roefs, A. y Jansen, A. (2006). Impulsivity in obese women. Appetite, 47, 253-256. Doi: 10.1016/j.appet.2006.05.008.

Noreña, D. y Sánchez, I. (2015). La impulsividad en los síndromes prefrontales: una perspectiva neuropsicológica. En J. L. Celma (Ed.): Bases teóricas y clínica del comportamiento impulsivo (pp. 71-92). Barcelona: Ediciones San Juan de Dios-Campus Docent. Recuperado de https://bibliosjd.files.wordpress.com/2015/02/bases_teoricas_y_clinica_comportamiento_impulsivo.pdf.

Organización Mundial de la Salud (2020). Obesidad y sobrepeso. Nota Descriptiva no. 311 [en línea]. Ginebra: OMS. Recuperado de https://www.who.int/es/news-room/fact-sheets/detail/obesity-and-overweight.

Organization for Economic Cooperation and Development (2017). Obesity update 2017. Paris: OECD. Recuperado de http://www. oecd.org/els/health-systems/Obesity-Update-2017.pdf.

Osuna R., I., Hernández P., B., Campuzano J., C. y Salmerón, J. (2006). Índice de masa corporal y percepción de la imagen corporal en una población adulta mexicana: la precisión del autorreporte. Salud Pública de México, 48(2), 94-103.

Pasco, J.A., Williams, L.J., Jacka, F.N., Brennan, S.L. y Berk, M. (2013). Obesity and the relationship with positive and negative affect. Australian \& New Zealand Journal of Psychiatry, 47(5), 477-482. Doi: 10.1177/0004867413483371.

Puhl, R.M., Luedicke, J. y Heuer, C.A. (2013). The stigmatizing effect of visual media portrayals of obese persons on public attitudes: does race or gender matter? Journal of Health Communication, 18(7), 805-826. Doi: 10.1080/10810730.2012.757393.

Robles, R., Varela, R., Jurado, S. y Páez, F. (2001). Versión mexicana del Inventario de Ansiedad de Beck: propiedades psicométricas. Revista Mexicana de Psicología, 18(2), 211-218. 
Schachar, R., Logan, G.D., Robaey, P., Chen, S., Ickowicz, A. y Barr, C. (2007). Restraint and cancellation: multiple inhibition deficits in attention deficit hyperactivity disorder. Journal of Abnormal Child Psychology, 35(2), 229-238. Doi: 10.1007/ s10802-006-9075-2.

Smith, E., Hay, P., Campbell, L. y Trollor, J.N. (2011). A review of the association between obesity and cognitive function across the lifespan: Implications for novel approaches to prevention and treatment. Obesity Reviews, 12, 740-755. Doi: 10.1111/j.1467-789x.2011.00920.x.

Verbruggen, F., Logan, G.D. y Stevens, M.A. (2008). STOP-IT: Windows executable software for the stop-signal paradigm. Behavior Research Methods, 40(2), 479-483. Doi: 10.3758/BRM.40.2.479.

Verdejo G., A. y Bechara, A. (2010). Neuropsicología de las funciones ejecutivas. Psicothema, 22(2), 227-235.

Volkow, N.D., Wang, G.J. y Baler, R.D. (2011). Reward, dopamine and the control of food intake: implications for obesity. Trends in Cognitive Sciences, 15(1), 37-46. Doi: 10.1016/j.tics.2010.11.001.

Winstanley, C.A. (2011). The utility of rat models of impulsivity in developing pharmacotherapies for impulse control disorders. British Journal of Pharmacology, 164(4), 1301-1321. Doi: 10.1111/j.1476-5381.2011.01323.x.

World Health Organization (2000). Obesity: preventing and managing the global epidemic. Report of a WHO Consultation. WHO Technical Report Series 894. Geneva: WHO.

Yuan, J., He, Y., Qinglin, Z., Chen, A. y Li, H. (2008). Gender differences in behavioral inhibitory control: ERP evidence from a two choice oddball task. Psychophysiology, 45, 986-993. Doi: 10.1111/j.1469-8986.2008.00693.x. 